\title{
Recurrence analysis of fractional-order Lorenz system
}

\author{
Andrzej Rysak ${ }^{1, *}$ and Magdalena Gregorczyk \\ ${ }^{1}$ Lublin University of Technology, Faculty of Mechanical Engineering, Nadbystrzycka 36, 20-618 \\ Lublin, Poland
}

\begin{abstract}
The fractional derivative is increasingly used in modeling of nonlinear systems. Fractional-order systems often give better fit to the experimental results, especially for the systems in which memory effects or hysteresis play a significant role. The paper presents numerical results obtained for Lorenz dynamical system, described by equations with the fractional derivative components. The impact of the fractional derivative terms on the system dynamics and stability is analyzed by using phase diagrams and recurrence plot analysis.
\end{abstract}

\section{Introduction}

The first mention of fractional calculus dates back 300 years. The idea of fractional derivative began to appear in conversations and correspondence between different scientists including Leibniz, L'Hospital and Bernoulli. Euler and Lagrange also considered this subject. The first attempts to define a fractional derivative were made by Laplace in 1812. The first use of it was in 1823 by Abel in the solution of tautochrome problem. Since then, interest in this subject has been constantly growing. One of the most important applications of partial calculations are found in chaos theory and nonlinear dynamical systems. Chaotic systems are very sensitive to changes in initial conditions and the approximation of the present state of the system does not approximate its future states. This very important property of chaotic systems was stated in the 1960s by Edward Lorenz [1] during his work on creating models for predicting meteorological forecasts.

In recent times, the use of fractional calculus in various fields of knowledge has been developed. One can find their application in bioengineering [2], heat transfer [3], energy harvesting [4, 5], nonlinear dynamics [6], and many others. In practice, differential equations with the fractional derivative are well suitable for modeling of many real systems. This means that in nature the 'fractional' dynamics is common and should be considered in the scientific research. Therefore, the influence of the fractional derivative on the dynamics of a nonlinear system is an interesting research topic, especially, as a method of studying real systems and phenomena. Among many methods that can be used in the study of stationarity of nonlinear systems dynamics, the investigations of their recurrencivity give promising results [7, 8]. Based on this, in the current paper, we focus on applying the recurrence quantification analysis (RQA) to study the influence of the fractional derivative on the nonlinear system dynamics.

Short description of recurrence plots (RP) and the RQA quantifiers is provided in Sec. 2. The Lorenz system is described in Sec. 3 by differential equations written for its original

\footnotetext{
*e-mail: a.rysak@pollub.pl
} 
form and in terms of the fractional derivative. In Sec. 4, the numerical results show how the selected RQA measures vary following the changes of the order of fractional derivative. The observed regularities and dependencies are discussed and summarize in the last section.

\section{Recurrence analysis}

The recurrence plots as a method to test the nonlinear systems was proposed by Eckmann [9], developed by Webber and Zbilut [10], Casdagli [11], later by Marwan et al. [12, 13] and others. In the first step, the RP method requires to move a considered time series to an m-dimensional embedding space [14]. However, in our case, for the considered system complete set of differential equations is known and the recursive analysis is carried out in a space with a dimension determined by the number of equations. In this paper it is assumed that changes of the system's dimension due to the fractional derivatives are small and all interesting phenomena are preserved when a trajectory of the system is projected onto a space with an integer number of dimensions. Next, for the vectorial time series being the solution of a nonlinear system, a distance matrix $R$ is defined which elements determine neighbors for each vector of the series. The elements $R_{i, j}^{\epsilon}$ of the matrix $R$ are defined using the Heaviside function

$$
R_{i j}^{\epsilon}=\Theta\left(\epsilon-\left\|\vec{Y}_{i}-\vec{Y}_{j}\right\|\right)
$$

where the Euclidean $L_{2}$-norm was used. The threshold parameter $\epsilon$ determines a proximity condition. Based on the sparse $R_{i j}^{\epsilon}$ matrix, some recurrence measures were calculated following their fundamental definitions and interpretations [15]. The basic measure of the RQA is recurrence rate $(\mathrm{RR})$

$$
R R(\epsilon, N)=\frac{1}{N^{2}-N} \sum_{i \neq j=1}^{N} R_{i, j}^{\epsilon}
$$

which is simply a measure of density of $R_{i j}^{\epsilon}$ non-zero (black) points in the RP matrix. The above formula excludes the identity points lying on the diagonal. In the assessment of system dynamics through the RP, the main role is played by diagonal and vertical lines. In Sec. 4 the results of two recurrence measures derived from the diagonal lines statistics (DET and $R A T I O)$ and two others calculated based on the vertical lines distribution (LAM and TT) are compared and analyzed. Diagonal lines correspond to repeats of some time series segments. The percent determinism (DET) is defined as the fraction of recurrence points that form diagonal lines

$$
D E T=\frac{\sum_{l=2}^{N-1} l H_{D}(l)}{\sum_{i \neq j=1}^{N} R_{i, j}}
$$

where $H_{D}(l)$ is the histogram of the lengths $l$ of the diagonal lines. The ratio (RATIO) is a measure derived from $D E T$ and equals

$$
R A T I O=\frac{D E T}{R R}=\left(N^{2}-N\right) \frac{\sum_{l=2}^{N-1} l H_{D}(l)}{\left(\sum_{i \neq j=1}^{N} R_{i, j}\right)^{2}} .
$$

Both measures are calculated without the identity points lying on the RP diagonal. The vertical lines (and the corresponding horizontal lines) occur when the system stays in a small area of the phase space for some time. Laminarity (LAM) and trapping time (TT) are defined as follows:

$$
L A M=\frac{\sum_{v=2}^{N} v H_{V}(v)}{\sum_{i, j=1}^{N} R_{i, j}}, \quad T T=\frac{\sum_{v=2}^{N} v H_{V}(v)}{\sum_{v=2}^{N} H_{V}(v)} .
$$


In the above equations, $v$ is the length of the vertical lines and $H_{V}(v)$ is the histogram of the lengths of the vertical lines. Analogously to DET, LAM determines the relative number of points lying on vertical lines. TT equals to the mean time the system is trapped in a specific state.

\section{Lorenz system with fractional derivative}

The Lorenz system is one of the best-known nonlinear dynamical systems. In its standard form it is described by the following equations [1]:

$$
\begin{aligned}
& \dot{x}=-\sigma(x-y) \\
& \dot{y}=-x z+r x-y \\
& \dot{z}=x y-b z
\end{aligned}
$$

where we adopt $\sigma=10, r=28$, and $b=8 / 3$ following [16]. In parallel, Lorenz fractional counterpart is shown with the fractional derivative operator defined as

$$
{ }_{L} D_{t}^{q}=\frac{d^{q}}{d t^{q}}
$$

where $L$ means the memory length possible to use in fractional calculations when applying the short memory principle $[6,17]$. Introducing fractional derivatives to the Lorenz system gives modified set of equations usually written in a general form as

$$
\begin{aligned}
& { }_{L} D_{t}^{q 1} x=-\sigma(x-y) \\
& { }_{L} D_{t}^{q 2} y=-x z+r x-y \\
& { }_{L} D_{t}^{q 3} z=x y-b z .
\end{aligned}
$$

In these preliminary investigations we study the dynamics of the system when the fractional derivative appears only in the first equation. Therefore, the equalities $q 2=q 3=1$ and $q=q 1$ were assumed in the further analysis of the system. Thanks to this assumption, it will be possible to assess changes in the dynamics of the system due to the modification of the fractional order of one variable (in one dimension).

\section{Numerical results}

In our numerical studies we apply the Grünwald-Letnikov definition of the fractional derivative introduced by Anton Karl Grünwald in 1867 and Aleksey Letnikov in 1868. Detailed information on its definition and calculation method can be found in the subject literature [6]. Below are shown the results obtained for the system under study. To compare the variability of the system for different $q$ values both characteristic phase diagrams and time series are presented.

Nonlinear system solutions were obtained for the adopted time step $h$ and memory length $L$. The duration time $t_{D}$ in all calculations was $2000 \mathrm{~s}$. Since a small time step $h$ was used in numerical calculations, the density of points in obtained solutions was large, which is not optimal for recursive calculations and significantly increases the size of the $R$ matrix. To extend the scope of recursive calculations, the density of time series points were reduced numerically. The value of the threshold parameter $\epsilon$ for which the RR value was at the level of a few percent was assumed. The value of $\epsilon$ is constant for the system, therefore the RR fluctuates in individual solutions following changes in any of its parameters or conditions. In 
a)
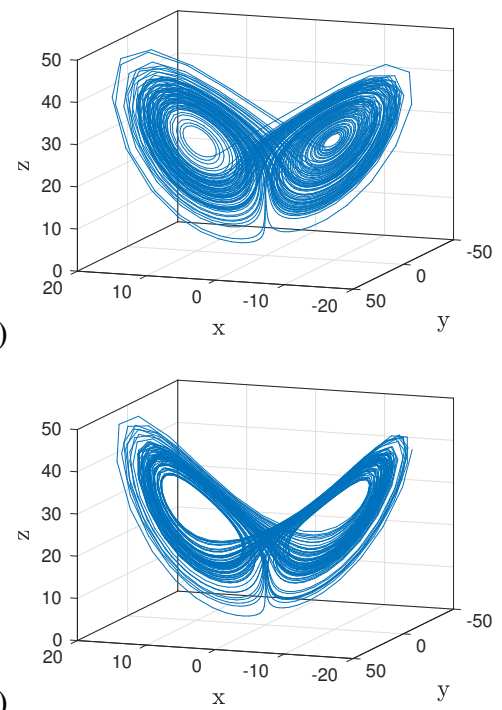

b)

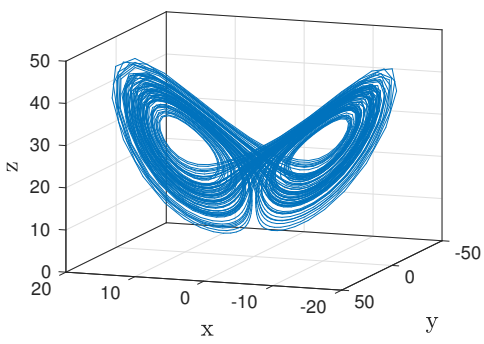

d)

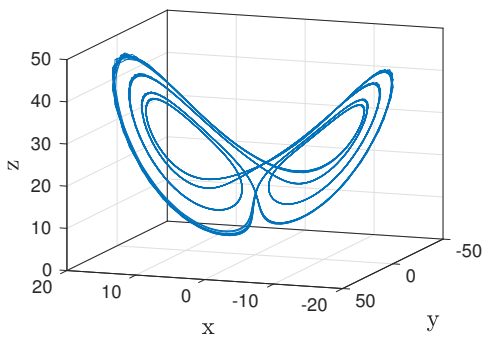

Figure 1. Phase diagrams obtained for the Lorenz system. $x(t), y(t), z(t)$ - solutions of the set (Eq. 8) for the values of $q$ equal: a) 1.0, b) 1.125 , c) 1.175 , and d) 1.2

a)
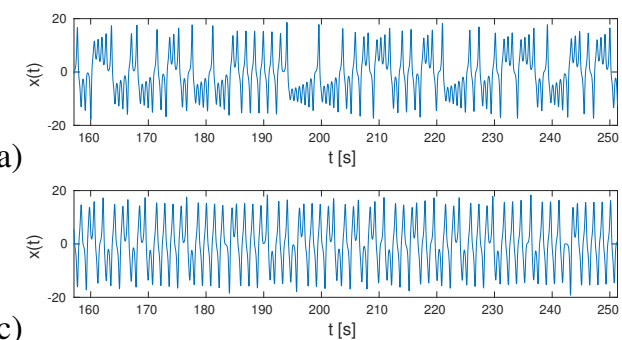

b)

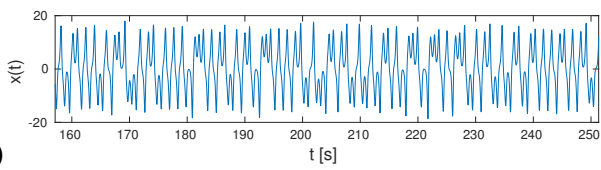

d)

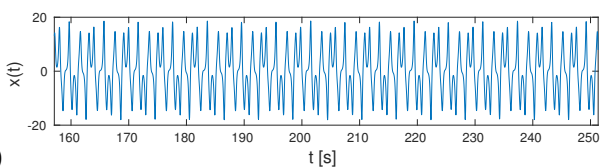

Figure 2. Time series corresponding to respective phase plots shown in Figure 1. $x(t)$ - solution of the first equation of the set (Eq. 8) for the values of $q$ equal: a) 1.0, b) 1.125, c) 1.175 , and d) 1.2

each case, recurrence measures were calculated for the matrix of $20000 \times 20000$ time points. The following parameters have been used in the calculations for this system: $L=2000$, $h=\pi / 1000$, and $\epsilon=0.5$.

There are two characteristic types of solutions in the results obtained for the Lorenz system. For $q>=1.2$ we get a complex, but periodic solution (Fig. 1d). For $q<1.2$ the solutions are chaotic, but they evolve with the change in its value (Fig. 1a-c). For the solutions presented on phase diagrams in Figure 1, the corresponding time series are shown in Figure 2. They indicate the evolution of the time variation of the first coordinate of the system (8). Based on the equations (3-5), the recurrence measures were calculated for $q$ varying from 0.9 to 1.3 (Figure 3). Each of them changes individually with $q$, reflecting to specific changes in the dynamics of the system. Figure 3 a shows that the trend of decreasing $D E T$ value with the increasing of $q$ is imposed by a rapid increase when the system goes to periodicity ( $q=1.2)$. The RATIO is sensitive mainly to the transition to periodicity. LAM indicates peak in the 
a)
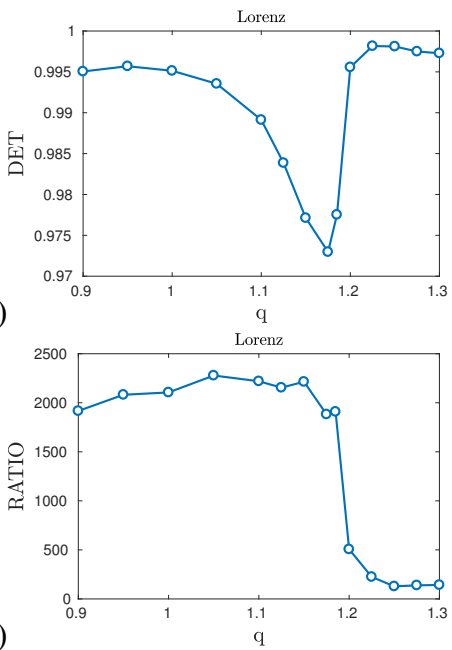

b)
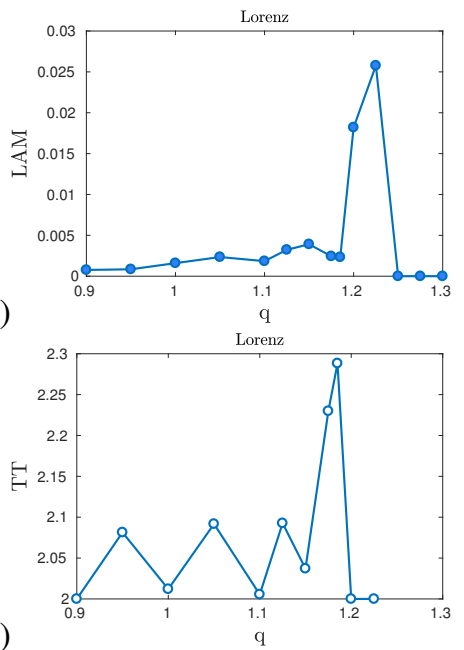

Figure 3. The Lorenz system. Values of recurrence measures as a function of the order of fractional derivative $q$

region of quasi-periodic fluctuations. In turn, the $T T$ fluctuates giving relatively weak peak just before transition to the periodicity.

\section{Conclusions}

In the present work several solutions of the fractional-order Lorenz system was analyzed for different values of $q$. The aim of the research was to test how the fractional derivative impacts on the recurrensivity of nonlinear system solutions. As it is known, the fractional derivative clearly changes the dynamics of nonlinear systems. Therefore, for the investigated system, the phase diagrams and time series were used to identify areas with special dynamics characteristics. By referring this information to the results of the recurrence research, it is possible to assess which aspects of a nonlinear dynamics influence individual recurrent measures.

Following the RATIO definition, it detects major changes in the system dynamics. In the Lorenz systems this measure rapidly changes its value when $q$ moves the system dynamics to the periodic mode. The measures derived from the vertical line statistics (LAM,TT) are of small values because of the lack of these lines. In spite of this, the variability of laminarity $(L A M)$ indicates sharp changes in the periodic region $(q>1.2)$, where the other measures have rather flat characteristics. The selection of appropriate values of embedding parameters and recurrence parameters results in a recurrence plot with points arranged mainly on diagonal lines. Under such circumstances, the DET value equals almost one, and the LAM is close to zero. Thick diagonal lines give higher value of the LAM measure due to the short lines statistics.

In our research, the DET value is approximately equal to one what confirms the correct selection of embedding and recursion parameters. But changes in the dynamics of the system involved by varying $q$ significantly modify its values, what is clearly seen in Figure 3a. As can be noticed in the figure, the value of this variable changes in a wide range of $q$. The most unique in this variable is its sensitivity to changes in the dynamics of the fractional-order system in the chaotic region. 
In summary, we can conclude that changes of recurrence measures reflect important features of the fractional-order nonlinear dynamical system. Particular recurrence measures are sensitive to the dynamics evolution of the investigated system in both chaotic $(D E T)$ and quasi-periodic $(L A M)$ regions, as well as clearly reflect the chaos - periodicity transition $(R A T I O)$. This preliminary results give hope for the potential use of the recursive analysis to determine the fractional order of the differential equations modeling the real dynamical systems tested experimentally.

This work was supported by the Polish National Science Centre under grant Agreement No. DEC2013/11/D/ST8/03308.

\section{References}

[1] E.N. Lorenz, J. atmos. Sci. pp. 120-141 (1963)

[2] R. Magin, Fractional Calculus in Bioengineering (Begell House Publishers, 2006)

[3] D. Sierociuk, A. Dzieliński, G. Sarwas, I. Petráš, I. Podlubny, T. Skovranek, Phil Trans R Soc A 371, 20120146 (2013)

[4] C.A. Kitio Kwuimy, G. Litak, C. Nataraj, Nonlinear Dynamics 80, 491 (2015)

[5] J. Cao, S. Zhou, D.J. Inman, Y. Chen, Nonlinear Dynamics 80, 1705 (2015)

[6] I. Petráš, Fractional Order Nonlinear Systems (Springer, Higher Education Press, Beijing and Springer-Verlag Berlin Heidelberg, 2011)

[7] A. Rysak, G. Litak, R. Mosdorf, in Recurrence Plots and Their Quantifications: Expanding Horizons, edited by J. Charles L. Webber, C. Ioana, N. Marwan (Springer Nature, Switzerland, 2016), Vol. 180, chap. 4, pp. 65-90

[8] G. Górski, G. Litak, R. Mosdorf, A. Rysak, Eur. Phys. J. Plus 131:111, 1 (2016)

[9] J.P. Eckmann, S.O. Kamphorst, D. Ruelle, Europhys. Lett. 5, 973 (1987)

[10] C.L. Webber, Jr., J.P. Zbilut, J. Appl. Physiology 76, 965 (1994)

[11] M. Casdagli, Phys. D 108, 12 (1997)

[12] N. Marwan, Ph.D. thesis, Universitaet Potsdam, Potsdam (2003)

[13] N. Marwan, M.C. Romario, M. Thiel, J. Kurths, Phys. Rep. 438, 237 (2007)

[14] F. Takens, in Dynamical Systems and Turbulence, edited by D.R.L. Young (SpringerVerlag, Berlin, 1981), pp. 366-381

[15] N. Marwan, C.L. Webber, Jr., in Recurrence Quantification Analysis, Understanding Complex Systems, edited by C.L. Webber, Jr., N. Marwan (Springer International Publishing, Switzerland, 2015), pp. 3-44

[16] J.C. Sprott, Chaos and Time-series Analysis (Oxford University Press, Oxford, UK \& New York, USA, 2003)

[17] I. Podlubny, Fractional Differential Equations (Academic Press, San Diego, 1999) 